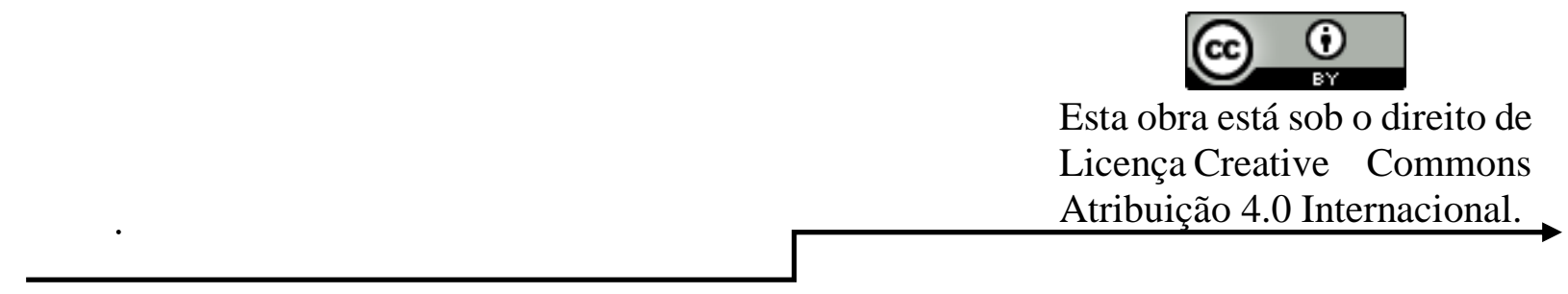

\title{
SUS: SAÚDE E CIDADANIA
}

\author{
Júlia Cláudia Tenório ${ }^{1}$ \\ Suzana Peixoto de Araújo ${ }^{2}$ \\ Marcelo Henrique Santos ${ }^{3}$ \\ Rafael Vital dos Santos 4 \\ Celsa Maria Calheiros de Emeri Tenório ${ }^{5}$
}

\section{RESUMO}

No Brasil, saúde e cidadania estão intensamente ligadas. Isso se dá a partir do princípio da participação popular do SUS, que permite que o cidadão comum tenha poder de influenciar na qualidade dos serviços do sistema de Saúde. O Sistema Único de Saúde (SUS) é um dos maiores e mais complexos sistemas de saúde pública do mundo, abrangendo desde o simples atendimento para avaliação da pressão arterial, por meio da Atenção Primária, até o transplante de órgãos, garantindo acesso integral, universal e gratuito para toda a população do país. Com a sua criação, o SUS proporcionou o acesso universal ao sistema público de saúde, sem discriminação. A atenção integral à saúde, e não somente aos cuidados assistenciais, passou a ser um direito de todos os brasileiros, desde a gestação e por toda a vida, com foco na saúde com qualidade de vida, visando a prevenção e a promoção da saúde. O Sistema Único de Saúde (SUS) é composto pelo Ministério da Saúde, Estados e Municípios, conforme determina a Constituição Federal. Cada ente tem suas co-responsabilidades. Na Metodologia deste artigo foram utilizadas pesquisas qualitativas com procedimentos e técnicas de revisão para o embasamento teórico do texto e pesquisas de artigos referentes ao tema estudado.

Palavras-chave: SUS e Cidadania; SUS; Cidadania; Sistema Único de Saúde.

\section{ABSTRACT}

In Brazil, health and citizenship are closely linked. This is based on the principle of popular participation by SUS, which allows ordinary people to have the power to influence the quality of the health system services. The Unified Health System (SUS) is one of the largest and most complex health systems public health in the world, ranging from simple care for blood pressure assessment, through Primary Care, to organ transplantation, ensuring full, universal and free access for the entire population of the country. With its creation, SUS provided universal access to the public health system, without discrimination. Comprehensive health care, and not only assistance care, has become a right for all Brazilians, since pregnancy and for life, focusing on

\footnotetext{
1 juliatenorio2017@gmail.com.br

2 prof_suzanereis@hotmail.com

3 drmarcelo_psf_odonto@yahoo.com.br

4 vittalbio@hotmail.com

5 celsatenorio@hotmail.com
} 
health with quality of life, aiming at prevention and health promotion. The Unified Health System (SUS) is composed of the Ministry of Health, States and Municipalities, as determined by the Federal Constitution. Each entity has its co-responsibilities. In the methodology of this article, qualitative research with review procedures and techniques was used for the theoretical basis of the text and research of articles related to the studied theme.

Key words: SUS and Citizenship; SUS; Citizenship; Health Unic. 


\section{INTRODUÇÃO}

A saúde da população brasileira comprova os amplos problemas estruturais em que o país está passando na realidade de nossos dias. Isto porque existe uma relação entre saúde e condições de vida como mostram vários autores. O presente artigo aborda esta inclusão e coloca o exercício da cidadania como estratégia para reverter esta situação apavorante em que se depara a população brasileira. Como forma de conseguir um exercício eficaz da cidadania, considera-se fundamental desenvolver um procedimento educativo consistente durante o período de educação formal que, além de estar voltado para profissionalização, deve atuar no desenvolvimento dos cidadãos.

Quando se fala em cidadania, na conjuntura do SUS, enquanto direito à saúde, é preciso observar além do acesso à assistência de saúde. Esse conceito de cidadania pode ser exercido e desfrutado pelo cidadão por ações de efetivo controle das decisões da saúde pública.

O Brasil, país em desenvolvimento, apesar de possuir um grande potencial sócio-econômico, tem que lidar com problemas estruturais de difícil solução. Isto acaba por refletir na saúde da população, pois grande parte desta não possui condições de vida adequadas. A relação entre saúde e qualidade de vida vem sendo discutida há algum tempo e vários trabalhos trazem como uma das principais causas das doenças existentes as más condições de vida, de trabalho, de habitação de uma população, preconizando reformas sanitárias, sociais e econômicas para o enfrentamento destas, como relata Buss (2000).

Minayo (2001) discutindo a problemática da saúde no Brasil observa que o quadro da saúde no país, incluindo a crescente violência urbana, está fortemente relacionado à grande desigualdade social existente. Além de outros fatores, também contribuem para a manutenção desta situação problemática a relação de domínio e submissão que ainda persiste no Brasil (MATUÍ, 2001).

A Promoção da Saúde surge então, como uma forte alternativa de atuação para a emancipação da população brasileira e melhoria das suas condições de vida e saúde. Suas estratégias de ação fundamentam-se na democracia, nas ações do Estado com políticas saudáveis, na intersetorialidade, na reorientação do setor saúde, propondo articulações e parcerias e, com relevância, no exercício da cidadania através da capacitação da população para a participação na formulação de políticas públicas saudáveis e nos processos de decisão (AERTS et al, 2004).

A Saúde - enquanto campo ideológico - não é neutra, expressa o resultado do confronto entre as políticas 
sociais e econômicas adotadas pelos governos não raras vezes ditadas por agrupamentos econômicos nacionais e internacionais e a pressão de movimentos sociais organizados. A área da Saúde veicula interesses e ideologias diversas, das quais identificamos, grosso modo, a disputa de dois grupos: um representado pelo pensamento neoliberal que defende o modelo privativista e elitista das práticas de saúde, com ênfase na utilização de tecnologia pesada; o outro construído à luz do socialismo, idealiza um sistema de saúde público, equânime, de alcance coletivo. Assim, a Saúde não deve ser vista como área isolada uma vez que sofre e exerce pressão dos e sobre outros setores da sociedade; é um campo de saber, portanto local de disputa de relações de poder (BRÊTAS, Oliveira, 1999).

No âmbito das políticas públicas, pode-se afirmar que a institucionalização do SUS representou uma conquista do setor

\section{METODOLOGIA}

A abordagem e a estratégia metodológica definida por este estudo procedem de revisão de literatura, cujas fontes de pesquisas são derivadas da utilização de artigos científicos nacionais e internacionais a partir de consultas às bases de dados do SUS (Sistema Único da Saúde) e de fontes de pesquisas derivadas da que compreende a saúde como um direito humano, resultado de mobilização e luta de atores e atrizes sociais e políticos comprometidos com os preceitos de igualdade de direitos e universalidade. No entanto, várias vezes ele tem sido alvo de políticas de cunho neoliberal que buscam rever os preceitos constitucionais visando destituir do Estado o dever de assegurar a saúde como um direito ao conjunto da população brasileira, pautadas na crença de que a saúde é uma mercadoria e, como tal, passível de comercialização. A Constituição Federal brasileira assegura que o seu financiamento é de coresponsabilidade da União, estados e municípios, sendo que a Lei 8.080/90 exige que os recursos financeiros destinados ao SUS sejam depositados em contas (Fundos de Saúde) em cada esfera de governo e que somente sejam movimentados sob a fiscalização dos Conselhos de Saúde (BRASIL, 1991).

utilização de artigos científicos e livros, cujos assuntos abordados nos mesmos referem-se ao tema tratado. Para o desenvolvimento deste trabalho serão utilizadas pesquisas qualitativas com procedimentos e técnicas de revisão para o embasamento teórico do texto e pesquisas de artigos referentes ao tema estudado.

O método da análise de conteúdo foi utilizado para a análise qualitativa, trata-se de um conjunto de técnicas que visa obter, 
por metodologia ordenada e objetiva, a explicitação e sistematização do conteúdo de documentos ou mensagens, por meio da construção de indicadores que permitam a inferência de conhecimentos relativos às condições de produção dessas mensagens.

\section{RESULTADOS E DISCUSSÕES}

\section{SUS e cidadania}

No tocante ao exercício de nossa cidadania, é evidente que com dificuldades locais, avançamos em termos de participação e controle social, mas ainda temos e sofremos muitas interferências de ordem política partidária e econômica que comprometem este exercício. Mesmo identificando e considerando os avanços em todos estes anos de existência, é fato que o SUS é ainda um projeto que esta em disputa na sociedade brasileira, e seus princípios necessitam ainda serem efetivados em toda sua magnitude. Lamentavelmente muitos governantes e parlamentares, aqueles que nós elegemos e colocamos em postos chaves da administração publica sejam nos municípios, nos estados e na união entendem e afirmam que o SUS é um sistema de saúde para os pobres, e a classe média cada vez se distancia mais do SUS no dia a dia, mas o utiliza até com privilégios quando se vê sem alternativas por parte dos grandes grupos da Saúde Suplementar que se verificarmos com lupa hoje ela concorre
A coleta das informações foi obtida por meio de pesquisas efetuadas dos teóricos mencionados nas referências bibliográficas e foi realizada e construídos em conformidade com as normas da ABNT.

diretamente com o SUS (OLIVEIRA, 2019).

Ainda conforme Oliveira, em sua analise da Conjuntura Política do SUS para esta digamos breve analise de conjuntura, necessitamos também reconhecer que o SUS enfrenta dilemas que precisam ser superados, para que possa avançar mais e se consolidar, garantindo assim, um atendimento de qualidade para toda a população conforme pudemos verificar nas várias questões elencadas recentemente no Seminário Nacional de Atenção Primária e no de Relação Publico x Privado, importante resgatar que estamos falando e reiterando o que já demanda dos Relatórios das ultimas Conferencias de Saúde.

Neste sentido o que temos: $O$ subfinanciamento, um modelo de atenção ainda voltado para a doença e não para a saúde, por exemplo, que de certa formar estão relacionados há um conjunto de fatores sociais e econômicos, que podem e devem ser superados se definitivamente contarmos com os sujeitos político que façam a sua defesa nos mais variados 
espaços e que não usem o SUS como moeda de troca ou em período das eleições partidárias onde vemos muitos falando do SUS com propostas boas, mas muitas absurdas. Esta é a analise de conjuntura que esta posta, mas que não se encerra aqui temos ainda que: Fortalecer a participação e o controle social sobre todas as instâncias e os agentes que fazem parte do SUS, fortalecendo seu atores/sujeitos às organizações da sociedade civil e os laços políticos que garantem a sustentabilidade das ações em saúde (OLIVEIRA, 2019).

Temos reconhecida acumulação histórica de formulações e proposições para ampliação dos debates e sua consistência com vistas aos pactos sociais. Além da importante proposição apresentada em setembro de 2015, há também o 'Manifesto Brasil-Nação', de março de 2017, com 172 subscrições originais, conhecido como Bresser-Pereira (PEREIRA, 2017), assim como o 'Plano Popular de Emergência', de maio de 2017, com 76 proposições sistematizadas em 10 itens da Frente Brasil Popular, além de outras que também poderiam ter acontecido com determinação após o mensalão ou após as mobilizações de 2013. Sem descuidar do aqui e agora das mobilizações e pactuações necessárias possíveis, tornou-se mais do que nunca inabdicável e inadiável a construção de objetivos e pactos para médio prazo que venham orientar o que fazer no prazo curto.
Já está insustentável o tensionamento do processo produtivo e da esmagadora maioria da população nas últimas décadas, devido à poderosa sucção da riqueza produzida pelo 'buraco negro' da acumulação financeira especulativa. Além do destino de mais de $50 \%$ do Orçamento Geral da União para os rentistas da dívida pública que retrai o investimento público no desenvolvimento, o investimento privado nas empresas é inibido pelos juros exorbitantes dos empréstimos pelos bancos privados e agências financeiras; e nas empresas maiores, intensificou-se a aplicação dos lucros no mercado financeiro inclusive em títulos da dívida pública, caindo o reinvestimento na empresa (SANTOS, 2017).

Ora, a esmagadora maioria da sociedade depende direta e exclusivamente do processo produtivo, formal ou informal: o trabalho assalariado e por produção, presencial ou não, autônomo, micro, média e grande empresa etc. São os trabalhadores miseráveis e sobreviventes com renda abaixo de 1 salário mínimo $(6,6 \%$ da população), a massa de trabalhadores pobres com renda 1 a 2 salários mínimos (23,2\%), trabalhadores classe média baixa com 2 a 5 salários mínimos (46,2\%), trabalhadores classe média média com 5 a 10 salários mínimos (15\%), totalizando até aqui $91 \%$ da população, aos quais podem ser acrescentados parte dos trabalhadores da 
classe média alta, por volta de $5 \%$, restando 3 a $4 \%$ de parte da classe média alta e elite que dependem do processo especulativo com descomunal e crescente concentração da renda (FRENTE BRASIL POPULAR, 2017).

\section{SUS e o setor da saúde}

A Saúde - enquanto campo ideológico - não é neutra, expressa o resultado do confronto entre as políticas sociais e econômicas adotadas pelos governos não raras vezes ditadas por agrupamentos econômicos nacionais e internacionais e a pressão de movimentos sociais organizados. A área da Saúde veicula interesses e ideologias diversas, das quais identificamos, grosso modo, a disputa de dois grupos: um representado pelo pensamento neoliberal que defende o modelo privativista e elitista das práticas de saúde, com ênfase na utilização de tecnologia pesada; o outro construído à luz do socialismo, idealiza um sistema de saúde público, equânime, de alcance coletivo. Assim, a Saúde não deve ser vista como área isolada uma vez que sofre e exerce pressão dos e sobre outros setores da sociedade; é um campo de saber, portanto local de disputa de relações de poder (Brêtas, Oliveira, 1999).

No âmbito das políticas públicas, pode-se afirmar que a institucionalização do SUS representou uma conquista do setor que compreende a saúde como um direito humano, resultado de mobilização e luta de atores e atrizes sociais e políticos comprometidos com os preceitos de igualdade de direitos e universalidade. No entanto, várias vezes ele tem sido alvo de políticas de cunho neoliberal que buscam rever os preceitos constitucionais visando destituir do Estado o dever de assegurar a saúde como um direito ao conjunto da população brasileira, pautadas na crença de que a saúde é uma mercadoria e, como tal, passível de comercialização.

Indubitavelmente o SUS é uma política de Estado, pública - não gratuita -, custeada por meio dos impostos recolhidos pelo governo. A Constituição Federal brasileira assegura que o seu financiamento é de co-responsabilidade da União, estados e municípios, sendo que a Lei 8.080/90 exige que os recursos financeiros destinados ao SUS sejam depositados em contas (Fundos de Saúde) em cada esfera de governo e que somente sejam movimentados sob a fiscalização dos Conselhos de Saúde (Brasil, 1991).

O Direito a Saúde, trata-se de um dos principais direitos conhecidos no Brasil, tendo importante destaque na $\mathrm{CF}$, que define saúde como um direito social fundamental, de caráter universal, que deve ser assegurado pelo Estado e efetivado por intermédio de políticas sociais e econômicas. 
São inúmeras as políticas públicas voltadas à promoção e garantia dos direitos mencionados na Constituição Federal. Iremos destacar no presente estudo três políticas presentes no cotidiano da população Brasileira a Política Nacional de Assistência Farmacêutica, a Política Nacional de Nutrição, e a política Nacional de Humanização da Atenção e Gestão no Sistema Único de Saúde (HumanizaSUS) bastante difundidas no SUS e que ainda acarretam cotidianamente a busca efetiva da cidadania, deixando clara a existência de problemas na alocação financeira dos recursos na administração pública.

David Diniz Dantas ao analisar a justiça brasileira e a necessidade de sua humanização, comenta que:

Não adianta incluir na Constituição princípios lindos de justiça social, dignidade da pessoa humana, proteção aos pobres, solidariedade, se eu não os concretizo, se não os trago para o discurso judicial, se continuo aplicando o legalismo formal (DANTAS, 2007).

A Lei $\mathrm{n}^{\circ} 8.080$ de 19 de setembro de 1990, reafirma a saúde como um direito fundamental do ser humano, devendo o Estado prover as condições indispensáveis para seu exercício integral, através da reformulação e execução de políticas econômicas e sociais (DIÁRIO OFICIAL DA UNIÃO, 2019).
A saúde de uma população não depende apenas dos serviços de saúde e do uso dos medicamentos. Entretanto, é inegável sua contribuição e a importância do mesmo no cuidado à saúde. Como uma ação de saúde pública e parte integrante do sistema de saúde, a Assistência Farmacêutica é determinante para a resolubilidade da atenção e dos serviços prestados em saúde e envolve a alocação de grande volume de recursos públicos (CONASS, 2011).

Segundo A Política Nacional de Assistência Farmacêutica oferta ações e serviços de saúde essencial à população, com regulamentações definidas e financiamento tripartite, com o direcionamento de proporcionar ao usuário um serviço de qualidade.

A Política Nacional de Medicamentos (PNM), publicada pela Portaria GM/MS n. 3916, em 1998 tem como propósito "garantir a necessária segurança, eficácia e qualidade dos medicamentos. A promoção do uso racional dos medicamentos e o acesso da população àqueles medicamentos essenciais (BRASIL, 1998).

Essa política vem fortalecer os princípios e diretrizes constitucionais, permitindo aos gestores melhores condições da assistência à saúde da população e como parte essencial da Política Nacional de Saúde, constitui um dos elementos 
fundamentais para a efetiva implementação de ações capazes de promover a melhoria das condições da assistência à saúde da população.

Dentre as condições indispensáveis, no tocante a este estudo, destaca-se também a Política Nacional de Alimentação e Nutrição, recentemente atualizada pela Portaria $\mathrm{n}^{\circ} 2.715$, de 17 de novembro de 2011, segundo a qual, para a afirmação plena dos potenciais de crescimento e desenvolvimento do ser humano, a alimentação e nutrição são requisitos básicos, necessários para uma vida com qualidade e garantia de cidadania (MINISTÉRIO DA SAÚDE, 2011).

A Constituição brasileira de 1988 menciona o direito à alimentação no rol dos Direitos Sociais, porém enfatiza o princípio da dignidade da pessoa humana já em seu artigo $1^{\circ}$, inciso III, como um dos princípios da República Federativa do Brasil. Ocorre que só se pode falar em "dignidade da pessoa humana" se seus direitos fundamentais forem, de fato, plenamente assegurados (SENADO FEDERAL, 2019).

O Encontro Nacional de Segurança Alimentar e Nutricional, promovido pelo Conselho Nacional de Segurança Alimentar e Nutricional (CONSEA), em Brasília (2006), realizou um balanço das iniciativas governamentais, constatando que, apesar dos avanços obtidos, a segurança alimentar e nutricional da população brasileira ainda carece de ações prioritárias do Poder Público (LEÃO,2017).

Apesar de a saúde ser reconhecida constitucionalmente como dever do Estado, o fornecimento de alimentos especiais por intermédio do SUS ainda demanda uma trajetória longa e cansativa na maior parte dos estados brasileiros. Uma das justificativas do Estado para a não garantia desse direito é a indisponibilidade de recursos financeiros e a falta de pactuação, porém, as iniquidades em saúde relacionadas aos recursos vão além de sua escassez, pois, no Brasil, grupos mais vulneráveis são diretamente afetados com a má gestão na distribuição desses recursos (SISSON, 2007).

A Política Nacional de Humanização - HumanizaSUS (PNH) foi criada pelo Ministério da Saúde, em 2003, a partir do reconhecimento de experiências inovadoras e concretas que compõem um "SUS que dá certo".

Há um pouco mais de uma década a PNH fomenta mudanças na atenção e na gestão ao convidar os sujeitos envolvidos a repensar e intervir no cotidiano da saúde pública brasileira. É uma iniciativa que busca provocar mudanças em modelos de gestão e de atenção, valorizando os diferentes sujeitos que participam do processo de produção de saúde: usuários, trabalhadores e gestores (MARTINS, 2017). 
Conforme, Nelson Santos (2010) "Ninguém conhece todo o SUS", essa ideia, vinda de um dos formuladores do SUS e grande defensor da saúde universal brasileira, enfatiza a complexidade com as muitas nuances da experiência em andamento no país. O SUS é uma produção coletiva, e seus inúmeros entraves e riquezas só podem ser compreendidos quando olhados por muitos de nós. Partimos da proposta de Santos e atrevemo-nos a afirmar que a mesma serve para a $\mathrm{PNH}$, assim como para outras políticas que agregam sujeitos ao primar pela produção de redes e agenciamentos no SUS.

O HumanizaSUS é mais do que a soma do Núcleo Técnico e da Coordenação Nacional que estão localizados em Brasília, assim como dos consultores regionais contratados pelo Ministério da Saúde. É uma política que buscar agregar profissionais e apoiadores em prol da implementação de um sistema humanizado que possibilite a reflexão e o debate entre as relações de contraste e distinção entre as diversas realidades vivenciadas oferecendo a possibilidade de influenciar e ser influenciado em seu cotidiano, ampliando o entendimento dos autores envolvidos e a busca e efetivação da cidadania (SANTOS, 2017).

O envolvimento de vários segmentos da sociedade possibilita o compromisso da humanização do SUS fortalecendo a autonomia e protagonismo dos sujeitos, de corresponsabilidade entre eles, de solidariedade dos vínculos estabelecidos, dos direitos dos usuários e da participação coletiva no processo de gestão (VERDI, 2019).

O HumanizaSUS é mais do que a soma do Núcleo Técnico e da Coordenação Nacional que estão localizados em Brasília, assim como dos consultores regionais contratados pelo Ministério da Saúde. Na gestão federal há os consultores, grupo contratado pelo Ministério da Saúde, que, dentre outras tarefas, apoiam as ações de humanização nos territórios para os quais são referência (SANTOS, 2017).

\section{Serviços do SUS}

Fazem parte do SUS os centros e postos de saúde, hospitais - incluindo os universitários, laboratórios, hemocentros, bancos de sangue, além de fundações e institutos de pesquisa, como a Fundação Oswaldo Cruz e o Instituto Vital Brazil. O sistema abrange desde a prevenção a procedimentos mais complexos como transplante de órgãos. O SUS é destinado a todos os cidadãos e é financiado com recursos arrecadados através de impostos e contribuições sociais pagos pela população e compõem os recursos do governo federal, estadual e municipal (BLOG. SAÚDE. GOV, 2020). 
Os números dos SUS surpreendem. Confira abaixo alguns dados:

- Em 2011, 145 milhões de pessoas dependiam exclusivamente do Sistema Único de Saúde no Brasil.

- Através do SUS é garantida assistência à urgência e à emergência nas ruas, por meio do SAMU.

- O sistema de saúde realiza, por ano, 3,2 bilhões de procedimentos ambulatoriais e 500 milhões de consultas médicas.

- O SUS tem a maior rede de bancos de leite humano do mundo.

- O SUS tem mais de 100 milhões de pessoas cobertas na atenção básica.

- O SUS é o sistema de saúde no mundo que mais faz transplantes totalmente gratuito. Em 2012, o Brasil realizou mais de 24 mil

\section{CONCLUSÃO}

Concluindo, nosso sistema público de saúde vem cumprindo um papel determinado pela estratégia neoliberal global de outro modelo denominado "Cobertura Universal de Saúde”, um arranjo público-privado que confronta os melhores sistemas públicos de saúde do mundo e nossas diretrizes constitucionais. Mesmo assim, o SUS foi a política pública mais avançada, com militância mais militante e politizada desde a luta contra a ditadura e os debates constitucionais. Ainda transplantes totalmente cobertos, gratuitos e públicos.

- São oferecidos gratuitamente 800 tipos de remédios nos hospitais e nos postos de saúde.

- 16,4 milhões de brasileiros fizeram ou fazem o tratamento de hipertensão, diabetes ou asma pegando remédios gratuitos.

- O governo está reformando e ampliando 20 mil Unidades Básicas de Saúde (UBS), sendo 10 mil já com obras contratadas, e está construindo 5.000 novas unidades.

83,4 mil pacientes são mantidos em serviços de diálise na rede pública de saúde, sendo que $90 \%$ desse total fazem hemodiálise.

- Noventa por cento do mercado de vacinas no Brasil é feito pelo Sistema Único de Saúde (BLOG. SAÚDE. GOV, 2020).

assim, o SUS ampliou a cobertura de todos os serviços públicos para a metade mais pobre da população anteriormente descoberta.

Portanto, o que esse estudo procura mostrar é que para uma população atingir uma boa condição de saúde e qualidade de vida, considerando a relação existente entre ambas, é necessário muito mais do que um atendimento de saúde, tratamento médico ou fornecimento de medicamentos e outros. A capacitação da população para o exercício da cidadania, isto é, para participar nas decisões que envolvem sua 
vida são essenciais, como propõe a Promoção da Saúde. Com um grande papel para o cumprimento desta meta, aparece a Educação e dentro desta, a escola tendo como responsabilidade não só o ensino formal, mas também a formação de cidadãos conscientes de seus direitos e deveres engajados na participação social.

É preciso abrir novos horizontes para a busca de experiências de gestão/organização que deram bons

\section{REFERÊNCIAS}

AERTS D et al. Promoção de Saúde: a convergência entre as propostas da vigilância da saúde e da escola cidadã.

Cad. Saúde Pública; 2004. 20 (4): 10201028.

\section{BLOG. SAÚDE. GOV. Conheça o} Sistema Único de Saúde (SUS).

Acessado em 20/08/2020. Disponível em:

<http://www.blog.saude.gov.br/index.php/ servicos/32814-conheca-o-sistema-unicode-saude-sus>.

\section{BRASIL. Constituição da República}

Federativa do Brasil. Brasília (DF), 1988. BRASIL. Portaria GM/MS no 3916 de 30 de outubro de 1998. Aprova a Política Nacional de Medicamentos. Disponível em http://bvsms.saude.gov.br/bvs/saudelegis/g m/1998/prt3916_30_10_1998.html. Acesso em 20/08/20.

BRASIL. Conselho Nacional de Secretários de Saúde. Assistência Farmacêutica no SUS. Brasília: DF, 2011. Disponível em http://www.conass.org.br/bibliotecav3/pdfs /colecao2011/livro_7.pdf. Acesso em $10 / 08 / 20$. resultados em diversos pontos do país. Difundir conhecimentos positivos, reacender novas esperanças e bom ânimo, desde a classe política, gestores, passando pelo corpo de profissionais técnicos e de atividades relacionadas, até os usuários desses serviços, tanto da esfera pública quanto privada. Afinal, o SUS é o sistema de saúde vigente no país e fazer com que ele dê certo é responsabilidade de todos.

BRÊTAS, A. C, OLIVEIRA E. M.

Interseções entre as áreas de conhecimento da gerontologia, da saúde e do trabalho: questões para reflexão. Saúde e Sociedade, São Paulo. 1999. ago/dez; 8(2): 59-82.

BUSS PM. Promoção da Saúde e Qualidade de Vida. Ciência e Saúde Coletiva-Abrasco. 2000; 5(1): 163-177.

CONSELHO FEDERAL DE MEDICINA. Opinião dos brasileiros sobre o atendimento na área de saúde 2018. Acessado em 03/09/2020. Disponível em: <https://portal.cfm.org.br/images/PDF/data folha_sus_cfm2018.pdf $>$.

DANTAS, D. D. A humanização da justiça. Revista ISTOÉ, no 1804 , 5/5/2004.

LAVOR, Adriano De. Um padrão bem pouco saudável. FIOCRUZ, Revista RADIS n ${ }^{\circ}$ 56, Rio de Janeiro, abr/2007.

DIÁRIO OFICIAL DA UNIÃO. Lei n. 8080 de 19 de setembro de 1990. Dispõe sobre as condições para a promoção, proteção e recuperação da saúde, a organização e o funcionamento dos 
serviços correspondentes e das outras providências.

\section{FRENTE BRASIL POPULAR. Plano} Popular de Emergência 2017. Disponível em:

<http://agenciabrasil.ebc.com.br/sites/_age nciabrasil2013/files/files/Plano_Popular_d e_Emergencia.pdf $>$. Acesso em: 08/08/2020.

\section{LEÃO, M. M. III Conferência Nacional} de Segurança Alimentar e Nutricional. Documento Final, 2007, p. 24. Acesso em: 10/08/2020. Disponível em:

<http://www.mds.gov.br/webarquivos/publ icacao/seguranca_alimentar/DHAA_SAN. pdf $>$.

\section{MARTINS CP, L C. Política}

HumanizaSUS: Ancorar um navio no espaço. Botucatu: 2017; 21(60):13-22. Acessado em: 05/09/20. Disponível em: https://www.scielo.br/pdf/icse/v21n60/180 7-5762-icse-1807-576220150614.pdf.

\section{MATUÍ J. Cidadão e Professor em}

Florestan Fernandes. São Paulo:Cortez; 2001.

\section{MINAYO MC. Condiciones de Vida,} Desigualdad y Salud a Partir Del Caso Brasileño. In Briceño-Leon, R; Minayo, M.C.; Coimbra Jr., C. E. A. (coordenadores). Salud e Equidad: uma mirada desde las ciencias sociales. Fiocruz; 2001.p.55-71.

MINISTÉRIO DA SAÚDE. Secretaria de Atenção à Saúde. Departamento de Atenção Básica. Portaria $\mathbf{N}^{\circ}$ 2.715, de 17 de novembro de 2011 - Atualiza a Política Nacional de Alimentação e Nutrição. Brasília (Brasil): Ministério da Saúde; 2011.

\section{OLIVEIRA, J. M. Analise da Conjuntura}

Política do SUS. Conselho Nacional de Saúde. Acessado em: 08/08/2020. Disponível em:
$<$ http://conselho.saude.gov.br/web_plenari a/Docs/ANALISE_DA_CONJUNTURA POLITICA_DO_SUS.pdf>.

PEREIRA, B.;-PEREIRA, L. C. et al. Manifesto do Projeto Brasil 2017. Acessado em: 08/08/2020. Disponível em: <https://www.scielosp.org/article/sdeb/201 7.v41n113/353-364/>.

PLANALTO. (2006). Lei de segurança alimentar e nutricional. Acessado em: 10/08/2020. Disponível em: <http://www4.planalto.gov.br/consea/conf erencia/documentos/lei-de-segurancaalimentar-e-nutricional>.

SANTOS, NR. Conjuntura atual: instigando a busca de rumos e o que fazer. (2017) Acessado em: 08/08/2020. Disponível em: https://www.scielosp.org/article/sdeb/2017. v41n113/353-364/\#>.

SANTOS NR. Posfácio. In: L' Abbate S. Direito à Saúde: discursos e práticas na construção do SUS. São Paulo: Hucitec; 2010. p. 281-83.

\section{SAUDE. GOV. Política Nacional de} Alimentação e Nutrição. Acessado em: 10/10/2020. Disponível em: $<$ httpbvsms.saude.gov.brbvspublicacoespo litica_nacional_alimentacao_nutricao.pdf>

SAUDE. GOV. Sistema Único de Saúde (SUS): estrutura, princípios e como funciona. Acessado em: 07/08/2020. Disponível em:

$<$ http://www.saude.gov.br/sistema-unicode-saude $>$.

SENADO FEDERAL. Constituiçãa 1988. Constituição da República Federativa do Brasil. Brasília: Senado Federal; 1988. Acessado em 10/08/2020.

SILVESTRE, ROBERTA DE MIRANDA. Judicialização da saúde: estudo de caso sobre as demandas judiciais em um 
município de pequeno porte no sul do Estado do Piauí. Dissertação (mestrado) Fundação Getúlio Vargas, São Paulo, 2018. p 14. Acessado em: 10/08/2020 Disponível em:

http://bibliotecadigital.fgv.br/dspace/handl e/10438/26132

SISSON MC. Considerações sobre o programa de saúde da família e a promoção de maior equidade na política de saúde. Saúde

Sociedade. 2007. Acessado em:

10/08/2020. Disponível em:

$<$ https://www.fen.ufg.br/revista/v14/n1/pdf /v14n1a08.pdf $>$.
VALENTE, Flávio L. Schieck. Direito Humano à Alimentação: desafios e conquistas. São Paulo: Cortez, 2012, p. 4.

VERDI M, et al. Acolhimento e Humanização nas Práticas de Gestão e Atenção à Saúde de Pessoas Privadas de Liberdade. Universidade Federal de Santa Catarina, Florianópolis, 2 edição, 2019, p 65. Acessado em 05/09/20. Disponível em: $<$ https://www.unasus.ufsc.br Modo de acesso: www.unasus.ufsc.br 